\title{
Dyskinesia in Parkinson's disease: a clinical complication and an open scientific question
}

\author{
Manolo Carta ${ }^{1}$ Etienne Hirsch ${ }^{2}$
}

Published online: 16 July 2018

C) Springer-Verlag GmbH Austria, part of Springer Nature 2018

\begin{abstract}
About 60 years have passed since the pioneering work of Arvid Carlsson on the effect of L-DOPA in animal models of Parkinson's disease and the research on the effect of this drug still attracts much interest. It is of these days the sad news that Prof. Carlsson has passed away at the age of 95; we want to take the chance of this special issue entitled "Dyskinesia in Parkinson's disease: clinical features and experimental evidence", to celebrate his outstanding contribution to this research field.
\end{abstract}

Despite 60 years of research on Parkinson's disease, L-DOPA still represents the most effective treatment for alleviating motor symptoms, at least during the first few years of treatment. However, as the disease progresses, the efficacy of L-DOPA is reduced and the appearance of motor complications, particularly L-DOPA-induced dyskinesia (LID), represents a major burden.

Dyskinesia is not only a clinical complication for the management of Parkinson's disease, but also an intriguing topic of research, which engaged a vast community of researchers. Great contribution to the understanding of the mechanisms underlying this complication has been allowed, in the last 10-15 years, by the introduction of validated animal models of dyskinesia, mainly rodents and non-human primates. Here, we mainly focus on peak-dose dyskinesia, which is the type of dyskinesia that we can best mimic in the animal models.

Thus, this special issue aims at providing an updated overview on the dyskinesia field by collecting a series of

Manolo Carta

manolocarta@unica.it

Etienne Hirsch

etienne.hirsch@upmc.fr

1 Department of Biomedical Sciences, University of Cagliari, Cittadella Universitaria SP 8, 09042 Monserrato, Italy

2 Inserm U 1127, CNRS UMR 7225, Sorbonne Universités, Institut du Cerveau et de la Moelle épinière, ICM,

75013 Paris, France contributions from most of the recognized international experts in the field.

First, we discuss the clinical features, incidence, and risk factors (Truong et al.), as well as the therapeutic strategies aimed at minimizing the appearance of dyskinesia (Matarazzo et al.; Antonini and Nitu). Second, we present the animal models that have been used to clarify the mechanisms underlying the appearance of peak-dose dyskinesia (Tronci and Francardo; Sgambato and Tremblay). We then proceed discussing the alterations in the neuronal circuitry and dopaminergic transmission induced by the dopamine neuronal loss and replacement therapy (Cenci et al.; Fisone and Spigolon; Moratalla et al.).

The striatum is an area where several neuronal systems converge and contribute to the fine regulation of the motor function. Thus, we also discuss how the above alterations result in perturbation of the serotoninergic (Carta and Bjorklund; Bishop et al.; Pagano et al.), glutamatergic (Gardoni et al.), cholinergic (Perez et al.), and adenosinergic (Pinna et al.) transmissions, and how these systems contribute to altered synaptic plasticity (Picconi et al.) and appearance of abnormal movements, and might be possible targets for pharmacological treatments. Indeed, to date, only the NMDA receptor antagonist amantadine is used clinically to dampen dyskinesia (Perez-Loret and Rascol), but there is hope for the above pre-clinical evidence to be translated into new therapeutic strategies.

We conclude discussing the role of inflammation (Carta et al.), and the impulse control disorder that can also emerge from L-DOPA treatment and that may share common pathways with dyskinesia (Fernagut et al.).

We hope that this work can be of significant help not only for the scientific community in this research field, but also for all the people, students, or young researchers that are approaching this theme for the first time.

We are very grateful to all contributors and colleagues involved in the reviewing process. 